Homology, Homotopy and Applications, vol.4(2), 2002, pp.315-336

\title{
ON CONSTRUCTING RESOLUTIONS OVER THE POLYNOMIAL ALGEBRA
}

\author{
LEIF JOHANSSON, LARRY LAMBE AND EMIL SKÖLDBERG \\ ( communicated by Clas Löfwall) \\ Abstract \\ Let $k$ be a field, and $A$ be a polynomial algebra over $k$. \\ Let $I \subseteq A$ be an ideal. We present a novel method for com- \\ puting resolutions of $A / I$ over $A$. The method is a synthesis \\ of Gröbner basis techniques and homological perturbation the- \\ ory. The examples in this paper were computed using computer \\ algebra.
}

\section{To Jan-Erik Roos on his sixty-fifth birthday}

\section{Introduction}

Throughout this paper, let $k$ be a field and $A=k\left[x_{1}, \ldots, x_{n}\right]$ be the polynomial ring over $k$. Monomials $x_{1}^{\alpha_{1}} \cdots x_{n}^{\alpha_{n}}$ in $A$ will be denoted by $\mathbf{x}^{\alpha}$ where $\alpha=\left(\alpha_{1}, \ldots, \alpha_{n}\right) \in \mathbb{N}^{n}$, when it is convenient.

A total order on monomials that satisfies $u<v \Longrightarrow m u<m v$ and $1<m$, for all monomials $m, u, v \neq 1$ in $A$, is called a monomial order on $A$ in this paper. A term of a given polynomial is a monomial summand along with its coefficient. Given a monomial order, one can talk about the leading term, $\mathrm{LT}(p)$ of a given polynomial $p \in A$. For an ideal $I$ in $A$, the ideal generated by the leading terms of elements in $I$ will be denoted by $\operatorname{LT}(I)$. An element of $A / I$ will be denoted by the usual notation $a+I$, where $a \in A$.

The relation of divisibility in $A$ will be denoted by $\preceq$, i.e. $p \preceq q$ if and only if $p$ divides $q$. Thus, for monomials $\mathbf{x}^{\alpha} \preceq \mathbf{x}^{\beta}$ if and only if for all $1 \leqslant i \leqslant n$, one has $\alpha_{i} \leqslant \beta_{i}$.

The main result of this paper is the derivation of an algorithm which gives an explicit resolution of $A / I$ over $A$ by "perturbing" a resolution of $A / \operatorname{LT}(I)$ over $A$. This is done using homological perturbation theory $[\mathbf{4}],[\mathbf{1 0}],[\mathbf{1}],[\mathbf{1 1}],[\mathbf{1 9}],[\mathbf{2 1}]$ and Gröbner basis theory $[\mathbf{8}],[\mathbf{3}]$.

Second author partially supported by INTAS grant $99-1222$

Received December 20, 2001, revised February 25, 2002; published on July 12, 2002.

2000 Mathematics Subject Classification: ABC123

Key words and phrases: Homology, Homotopy, Homological Perturbation, Resolution, Polynomial

Ring, Gröbner Basis, Normal Form

(C) 2002, Leif Johansson, Larry Lambe and Emil Sköldberg. Permission to copy for private use granted. 


\section{Homological Perturbation Theory}

We will need to construct and manipulate explicit chain homotopy equivalences between certain chain complexes. There is a special theory behind doing this in a systematic way which is well suited for machine computations. It will be quickly reviewed in this section.

\subsection{Strong Deformation Retracts}

Let $X$ and $Y$ be chain complexes over $k, \nabla: X \longrightarrow Y, f: Y \longrightarrow X$ be chain maps and let $\phi: Y \longrightarrow Y$ be a degree one $k$-linear map such that $f \nabla=1_{X}$ and $d \phi+\phi d=1-\nabla f$. Thus, $f$ and $\nabla$ compose to the identity, but the composition the other way around is only chain homotopic to the identity. When these conditions hold, we say that this collection of data forms a strong deformation retraction (SDR) and we write

$$
X \underset{f}{\stackrel{\nabla}{\longleftarrow}}(Y, \phi) .
$$

Important for certain computations are the side conditions $[\mathbf{1 7}]$

$$
\phi^{2}=0, \quad \phi \nabla=0, \quad \text { and }, \quad f \phi=0 .
$$

In fact, these may always be assumed to hold: if the last two do not hold, replace $\phi$ by $\phi^{\prime}=D(\phi) \phi D(\phi)$ where $D(\phi)=\phi d+d \phi$ and the last two conditions will now hold with respect to $\phi^{\prime}$. If the first condition does not hold for $\phi^{\prime}$, replace it by $\phi^{\prime \prime}=\phi^{\prime} d \phi^{\prime}$ and all three conditions will hold for the chain homotopy $\phi^{\prime \prime}$. We will always assume that the side conditions hold when we talk about an SDR.

\subsection{The Perturbation Lemma}

Given the SDR (2.1) and, in addition, a second differential $d_{Y}^{\prime}$ on $Y$, let $t=$ $d_{Y}^{\prime}-d_{Y}$. The perturbation lemma, [4], [10], [1], [21] states that if we set $t_{n}=$ $(t \phi)^{n-1} t, n \geqslant 1$ and, for each $n$, define new maps on $X$,

$$
\begin{gathered}
\partial_{n}=d+f\left(t_{1}+t_{2}+\cdots+t_{n-1}\right) \nabla \\
\nabla_{n}=\nabla+\phi\left(t_{1}+t_{2}+\cdots+t_{n-1}\right) \nabla,
\end{gathered}
$$

and on $Y$ :

$$
\begin{aligned}
& f_{n}=f+f\left(t_{1}+t_{2}+\cdots+t_{n-1}\right) \phi \\
& \phi_{n}=\phi+\phi\left(t_{1}+t_{2}+\cdots+t_{n-1}\right) \phi,
\end{aligned}
$$

then in the limits, provided they exist, we have new SDR data

$$
\left(X, \partial_{\infty}\right) \underset{f_{\infty}}{\stackrel{\nabla_{\infty}}{\rightleftarrows}}\left(\left(Y, d_{Y}^{\prime}\right), \phi_{\infty}\right) .
$$

Note that the limits will certainly exist if $t \phi$ is nilpotent in each degree. Examples can be found in $[\mathbf{4}],[\mathbf{1 0}],[\mathbf{1 4}],[\mathbf{1 5}],[\mathbf{1 7}],[\mathbf{1 6}],[\mathbf{2 0}],[\mathbf{1 8}],[\mathbf{1 1}],[\mathbf{1 2}],[\mathbf{1 5}],[\mathbf{1 9}],[\mathbf{2 1}]$ and $[\mathbf{2 6}]$. 
Remark 2.1. In trying to set up a situation as above, it is convenient to think of the perturbation as transferring the new differential $d^{\prime}$ on the larger complex to the smaller one. In this sense, the data is said to be a transference problem and $t$ is called the initiator. This notation is from [1] and we will continue to use it here.

\subsection{Two Sided Data}

There are situations where complexes $X$ and $Y$ are chain homotopic, but there is no SDR involving them, of course. Consider data of the form

$$
(\psi, X) \stackrel{\nabla}{\longleftarrow}(Y, \phi)
$$

where $\nabla$ and $f$ are chain maps, and $\phi$ and $\phi$ are chain homotopies such that

$$
\nabla f=1_{Y}-D(\phi), f \nabla=1_{X}-D(\psi) .
$$

There are perturbation formulae for such two-sided data as well [1], [2], [15]. The situation will be encountered in this paper in Section 4.1, but we will manage to avoid having to use the more complicated two sided formulae in our main results.

\section{Resolutions}

For a module $N$ over $A$, we will generally consider resolutions of $N$ over $A$ of the form $X=A \otimes \bar{X}$ where $\bar{X}$ is free over $k[\mathbf{2 3}],[\mathbf{5}]$. Such complexes are called relatively free $[\mathbf{2 3}]$. Furthermore, it will be assumed that there is an explicit SDR

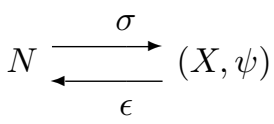

where $\epsilon$ is an $A$-linear map, but generally, $\sigma$ and $\psi$ are only $k$-linear. Here $N$ is given the zero differential. Note that in this context, $\psi$ is an explicit contracting homotopy.

Remark 3.1. It is standard terminology to call the elements of $\bar{X}$ above reduced elements. We follow this convention throughout the paper.

\subsection{The Taylor Resolution}

For a given set $\left\{m_{1}, \ldots, m_{r}\right\}$ of monomials and for any subset $J=\left\{j_{1}, \ldots j_{s}\right\} \subseteq$ $\{1, \ldots, r\}$, let $|J|=s, m_{J}=\operatorname{lcm}\left(m_{j_{1}}, \ldots, m_{j_{s}}\right)$ ("least common multiple"), and $J^{i}=J-\left\{j_{i}\right\}$. Let $E=E\left[u_{1}, \ldots, u_{r}\right]$ be the exterior algebra over $k$. $E$ has a $k$-basis given by $\left\{u_{J} \mid J \subseteq\{1, \ldots, r\}\right\}$ where for $J=\left\{j_{1}, \ldots j_{s}\right\}, u_{J}=u_{j_{1}} \ldots u_{j_{s}}$.

For a monomial ideal $M=\left(m_{1}, \ldots, m_{r}\right)$, the Taylor resolution [27] of $A / M$ over $A$ is given by the following $A$-linear differential $d$ on the free $A$-module $A \otimes$ $E\left[u_{1}, \ldots, u_{r}\right]$ :

$$
d\left(u_{J}\right)=\sum_{i=1}^{|J|}(-1)^{i-1} \frac{m_{J}}{m_{J^{i}}} u_{J^{i}} .
$$


This resolution will be denoted by $T$ throughout this paper. An explicit contracting homotopy for $T$ was given by Fröberg in [7] and is recalled here.

For a monomial $\mathbf{x}^{\alpha}$ and a basis element $u_{J}$ of $E$, let

$$
\iota\left(\mathbf{x}^{\alpha} u_{J}\right)=\min \left\{i \mid m_{i} \preceq \mathbf{x}^{\alpha} m_{J}\right\}
$$

Note that $\iota\left(\mathbf{x}^{\alpha} u_{J}\right) \leqslant j_{1}$.

Define a $k$-linear endomorphism of $T$ by

$$
\psi\left(\mathbf{x}^{\alpha} u_{J}\right)=\left[\iota<j_{1}\right] \frac{\mathbf{x}^{\alpha} m_{J}}{m_{\{\iota\} \cup J}} u_{\{\iota\} \cup J}
$$

where $\iota=\iota\left(\mathbf{x}^{\alpha} u_{J}\right)$, and $[p]$ is the Kronecker-Iverson symbol [9] which is zero if $p$ is false and one otherwise. The following will be used later.

Lemma 3.2. $\psi^{2}=0$.

Proof. If $\psi\left(\mathbf{x}^{\alpha} u_{J}\right) \neq 0$ and $\iota=\iota\left(\mathbf{x}^{\alpha} u_{J}\right)$, then $\iota\left(\frac{\mathbf{x}^{\alpha} m_{J}}{m_{\{\iota\} \cup J}} u_{\{\iota\} \cup J}\right)=\min \left\{i \mid m_{i} \preceq\right.$ $\left.\mathbf{x}^{\alpha} m_{J}\right\}=\iota\left(\mathbf{x}^{\alpha} u_{J}\right)=\iota$.

It is not true that $\psi$ vanishes on reduced elements in general as the following example shows. The example also shows the dependence of $\psi$ on the given order in which the monomials are listed.

Example 3.3. Let $A=k\left[x_{1}, x_{2}, x_{3}\right]$ and $M=\left(x^{3} y^{2}, x^{3} y z, x y^{5} z\right)$. Choosing the order $m_{1}=x^{3} y^{2}, m_{2}=x^{3} y z$, and $m_{3}=x y^{5} z$, one has $\psi(u)=0$ for all $u \in$ $E\left[u_{1}, u_{2}, u_{3}\right]$ unless $u=u_{1} u_{2}$ and $\psi\left(u_{1} u_{2}\right)=u_{1} u_{2} u_{3}$. The order $m_{1}=x y^{5} z, m_{2}=$ $x^{3} y z, m_{3}=x^{3} y^{2}$ however, gives $\psi(u)=0$ for all $u \in E\left[u_{1}, u_{2}, u_{3}\right]$.

The following lemma which is a straightforward consequence of "normal forms" $[\mathbf{8}],[\mathbf{3}]$ will be needed later.

Lemma 3.4. There is an explicit SDR

$$
A / M \underset{q}{\stackrel{\mathrm{nf}_{M}}{\rightleftarrows}}(T, \psi)
$$

where $q(a \otimes 1)=a+M$ and is otherwise zero, $\operatorname{nf}_{M}(a)$ is the normal form of a with respect to $M$ (using some monomial order - see the Remark below), and $\psi$ is the contracting homotopy just defined.

Remark 3.5. The calculation of normal forms is crucial to the algorithms presented in this paper. It depends on a given monomial order and a given set $G=$ $\left\{g_{1}, \ldots, g_{r}\right\} \subset A$. Here is a recursive algorithm for computing the normal form, denoted by $\operatorname{rem}_{B}(p)$ of a given polynomial $p$ :

if there exists a minimal $i$ such that $\operatorname{LT}\left(g_{i}\right) \preceq \operatorname{LT}(p)$, then

$$
\operatorname{rem}_{G}(p)=\operatorname{rem}_{G}\left(p-\frac{\mathrm{LT}(p)}{\mathrm{LT}\left(g_{i}\right)} g_{i}\right)
$$

and

$$
\operatorname{rem}_{G}(p)=\mathrm{LT}(p)+\operatorname{rem}_{G}(p-\operatorname{LT}(p))
$$


otherwise.

The Taylor resolution is not, in general, minimal. Various characterizations of the minimality of $T$ were given in [7].

\subsection{The Lyubeznik Resolution}

A subcomplex of the Taylor resolution which also is a resolution of the monomial module $A / M$ was given in [22]. This resolution will be denoted by $L$ and will be called the Lyubeznik resolution. Suppose that $M=\left\{m_{1}, \ldots, m_{r}\right\}$. For a given $I \subseteq\{1, \ldots, r\}$ and positive integer $s$ between 1 and $r$, let $I_{>s}=\{i \in I \mid i>s\} . L$ is generated by those basis elements $u_{I}$ which satisfy the following condition for all $1 \leqslant s<r$ :

$$
m_{s} \npreceq M_{I_{>s}} .
$$

Note that the construction of the Lyubeznik resolution depends on the chosen order of monomials. For example,

Example 3.6. Let $A=k\left[x_{1}, x_{2}, x_{3}\right]$ and $M=\left(x_{1} x_{3}, x_{1}^{2} x_{2}, x_{2} x_{3}^{2}\right)$. Consider the Lyubeznik resolution of $A / M$ with respect to the order $m_{1}=x_{1} x_{3}, m_{2}=x_{1}^{2} x_{2}, m_{3}=$ $x_{2} x_{3}^{2} . L$ has basis elements $u_{\{1\}}, u_{\{2\}}, u_{\{3\}}$ in degree 1 and $u_{\{1,2\}}, u_{\{1,3\}}$ in degree 2 and that is all.

If instead, the order $m_{1}=x_{1}^{2} x_{2}, m_{2}=x_{2} x_{3}^{2}, m_{3}=x_{1} x_{3}$ is chosen, the Lyubeznik resolution is equal to the Taylor resolution.

It is not hard to see that the basis elements of $L$ generate a subcomplex of $T$. The exactness can be proved by using the following lemma:

Lemma 3.7. The contracting homotopy $\psi$ of the Taylor resolution satisfies $\psi(L) \subseteq$ $L$.

Proof. Let $u_{I}$ be a basis element of $L, m=\mathrm{x}^{\alpha}$ be a monomial, and $\iota=\iota\left(m u_{I}\right)$. If $\iota=i_{1}, \psi\left(m u_{I}\right)=0$ and we are done. Thus suppose that $\iota<i_{1}$ so that $\psi\left(m u_{I}\right)=$ $\frac{m m_{I}}{m_{\{\iota\}} \cup I} u_{\{\iota\} \cup I}$. Let $1 \leqslant s<r$. If $s \geqslant \iota$, then clearly, $(\{\iota\} \cup I)_{>s}=I_{>s}$ and we are done by hypothesis. If $s<\iota,(\{\iota\} \cup I)_{>s}=I$ and $m_{s} \npreceq m_{I}$ by the minimality of $\iota$. Thus, $u_{\{\iota\} \cup I}$ is in $L$ and hence $L$ is invariant under $\psi$.

The following property will play an important role in what follows.

Lemma 3.8. $\psi(\bar{L})=0$.

Proof. Let $\iota=\iota\left(u_{I}\right)$. As in the proof above, if $\iota=i_{1}, \psi\left(u_{I}\right)=0$ and if $\iota<i_{1}$, $I_{>\iota}=I$. In the latter case, $m_{\iota} \preceq I_{>\iota}$, but that contradicts the hypothesis that $u_{I}$ is a basis element of $L$.

3.3. Splitting homotopies : Another View of the Lyubeznik Resolution

There are systematic ways to find smaller resolutions of a given resolution which are actually subresolutions. This is a special case of the following. 
Splitting homotopies were defined in [1]. The notion is recalled here. Let $Y$ be a chain complex over $k$. A splitting homotopy is a degree one map $\phi: Y \longrightarrow Y$ which satisfies

$$
\begin{aligned}
\phi \phi & =0 \\
\phi d \phi & =\phi .
\end{aligned}
$$

A given splitting homotopy gives rise to a strong deformation retract [1]

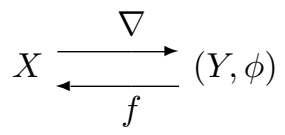

where $\nabla$ and $f$ are chain maps that satisfy

$$
\begin{aligned}
f \nabla & =1_{X} \\
\nabla f & =1-D(\phi) \\
\phi f & ==0 \\
\nabla \phi & =0 .
\end{aligned}
$$

To see this, set $\pi=1-D(\phi)$ and note that the conditions above imply that $\pi \pi=\pi$. If we take $X=\operatorname{im}(\pi)$, we have $Y=X \oplus \operatorname{ker}(\pi)$ and we can take $\nabla$ to be the inclusion of $X$ in $Y$ and take $f(x)=\pi(x)$. The converse is also true. See [1].

Given an explicit resolution $X=A \otimes_{k} \bar{X}$ of an $A$-module over $A$, we can thus seek $A$-linear splitting homotopies $\phi: X \longrightarrow X$ as a way of obtaining smaller resolutions. In fact, we have

Proposition 3.9. Let $T=A \otimes_{k} \bar{T}$ be the Taylor resolution, so that $\bar{T}$ has basis $\left\{u_{I}\right\}$ as before. Also let $\psi$ be the contracting homotopy (3.1). There is a splitting homotopy

$$
T \stackrel{\phi}{\longrightarrow} T
$$

which gives rise to $L$.

In fact, inductively define a linear map $T \stackrel{f}{\longrightarrow} T$ by requiring that $f$ is A-linear and satisfies

$$
f\left(u_{I}\right)=\psi\left(f\left(d u_{I}\right)\right)
$$

on reduced elements. Then we have an $S D R$

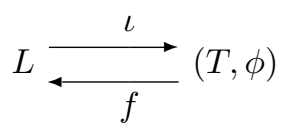

where $\iota$ is the inclusion.

Proof. Consider $L$ as a subcomplex of $T$. We know that the contracting homotopy $\psi$ takes $L$ to $L$ so we use it to construct the maps defined in Section 4.1. This gives exactly the recursive formula above and the formula

$$
\phi\left(u_{I}\right)=\psi\left(\phi\left(d u_{I}\right)+f\left(u_{I}\right)-u_{I}\right)
$$


on reduced elements for the $A$-linear homotopy $\phi$. It is straightforward to verify that this is actually an SDR and that $\phi$ is a splitting homotopy. Note that since $\psi$ actually vanishes on reduced elements by Lemma 3.8, the formula for $\phi$ above becomes $\phi\left(u_{I}\right)=\psi\left(\phi\left(d u_{I}\right)+f\left(u_{I}\right)\right)$ on reduced elements.

A related homotopy is given by the following construction. Define $\widehat{\psi}$ to be the unique $A$-linear extension of the map on $\bar{T}$ given by

$$
\widehat{\psi}\left(u_{I}\right)=\psi\left(u_{I}\right) .
$$

Clearly, $\widehat{\psi}$ is a degree one $A$-linear map and we have $\widehat{\psi} \widehat{\psi}=0$. We also have

\section{Proposition 3.10.}

$$
\begin{aligned}
\widehat{\psi} \widehat{\psi} & =0 \\
\widehat{\psi} d \widehat{\psi}\left(u_{I}\right) & =\widehat{\psi}\left(u_{I}\right)
\end{aligned}
$$

for all $I$.

Proof. We have

$$
\begin{aligned}
\widehat{\psi} d \widehat{\psi}\left(u_{I}\right) & =\widehat{\psi} d \psi\left(u_{I}\right) \\
& =\widehat{\psi}\left(\left(1-\psi d\left(u_{I}\right)\right)\right. \\
& =\widehat{\psi}\left(u_{I}\right)-\psi d\left(u_{I}\right)
\end{aligned}
$$

but

$$
\psi d\left(u_{I}\right)=\sum(-1)^{i} \psi\left(\frac{m_{I}}{m_{I^{i}}} u_{I^{i}}\right)
$$

and any term that is non-zero is of the form

$$
\frac{m_{I}}{m_{I^{i}}} \psi\left(u_{I^{i}}\right)=a u_{j I^{i}}
$$

where $j$ is the minimal index such that $m_{j} \mid \frac{m_{I}}{m_{I^{i}}} n_{I^{i}}$, i.e. $m_{j} \mid m_{I}=l c m\left(m_{1}, \ldots, m_{s}\right)$. Thus, $\widehat{\psi}\left(a u_{j I^{i}}\right)=\psi\left(u_{j I^{i}}\right)$, but $m_{j I^{i}}=l c m\left(m_{j}, m_{1}, \ldots, \hat{m}_{i}, \ldots, m_{s}\right)$ (where the $\hat{m}_{i}$ denotes omission) and so clearly $j=\iota\left(u_{j I^{i}}\right)$ from which it follows that $\psi\left(u_{j I^{i}}\right)=0$ by definition.

We have not extensively investigated the properties of this splitting homotopy in general, but note that sometimes it coincides with the splitting homotopy that gives the Lyubeznik resolution.

\subsection{The Bar Resolution}

Consider the two-sided bar construction $B(A, A)[\mathbf{2 3}]$, [5]. One has $B(A, A)=$ $A \otimes_{k} \bar{B}(A) \otimes_{k} A$ where $\bar{B}(A)=\sum_{n=0}^{\infty} \bar{B}_{n}(A)$,

$$
\begin{aligned}
& \bar{B}_{0}(A)=k \\
& \bar{B}_{n}(A)=\otimes^{n} \bar{A}, n>0,
\end{aligned}
$$

and $\bar{A}=\operatorname{coker}(\sigma)$ where $k \stackrel{\sigma}{\longrightarrow} A$ is the unit. The usual convention is to write $a \otimes$ $a_{1} \otimes \ldots a_{n} \otimes a^{\prime}$ as $a\left[a_{1}|\ldots| a_{n}\right] a^{\prime}$ and we will follow that convention. The differential 
in $B(A, A)$ is given by the $A$-linear map induced by

$$
\begin{aligned}
\partial\left(\left[a_{1}|\ldots| a_{n}\right] a^{\prime}\right) & =a_{1}\left[a_{2}|\ldots| a_{n}\right] a^{\prime} \\
& +\sum_{i=1}^{n-1}(-1)^{i}\left[a_{1}\left|\ldots a_{i} a_{i+1} \ldots\right| a_{n}\right] a^{\prime} \\
& +(-1)^{n}\left[a_{1}|\ldots| a_{n-1}\right] a_{n} a^{\prime}
\end{aligned}
$$

The $k$-linear map $B(A, A) \stackrel{s}{\longrightarrow} B(A, A)$ is defined by

$$
s\left(a\left[a_{1}|\ldots| a_{n}\right] a^{\prime}\right)=\left[a\left|a_{1}\right| \ldots \mid a_{n}\right] a^{\prime} .
$$

The map $A \stackrel{\sigma}{\longrightarrow} B(A, A)$ is given by

$$
\sigma(a)=[] a
$$

and the map $B(A, A) \stackrel{\epsilon}{\longrightarrow} A$ is given by

$$
\begin{aligned}
\epsilon\left(a[] a^{\prime}\right) & =a a^{\prime} \\
\epsilon\left(a\left[a_{1}|\ldots| a_{n}\right] a^{\prime}\right) & =0, n \geqslant 1 .
\end{aligned}
$$

With the zero differential in $A$, these maps form an SDR

$$
A \underset{\epsilon}{\stackrel{\sigma}{\longleftarrow}}(B(A, A), s) \text {. }
$$

For any right $A$-module $N$, one has a free $A$-complex given by $B(A, N)=$ $B(A, A) \otimes_{A} N$. As an $A$-module, note that

$$
B(A, N)=A \otimes_{k} \bar{B}(A) \otimes_{k} N .
$$

The differential is given by $\partial_{N}=\partial \otimes 1_{N}$. Thus,

$$
\begin{aligned}
\partial_{N}\left(a\left[a_{1}|\ldots| a_{n}\right] x\right) & =a a_{1}\left[a_{2}|\ldots| a_{n}\right] x \\
& +\sum_{i=1}^{n-1}(-1)^{i} a\left[a_{1}\left|\ldots a_{i} a_{i+1} \ldots\right| a_{n}\right] x \\
& +(-1)^{n} a\left[a_{1}|\ldots| a_{n-1}\right] a_{n} x
\end{aligned}
$$

for $a, a_{i} \in A$ and $x \in N$. Define $N \stackrel{\sigma_{N}}{\longrightarrow} B(A, N)$ by

$$
\sigma_{N}(x)=[] x,
$$

$B(A, N) \stackrel{\epsilon_{N}}{\longrightarrow} N$ by

$$
\begin{gathered}
\epsilon_{N}(a[] x)=a x \\
\epsilon_{N}\left(a\left[a_{1}|\ldots| a_{n}\right] x=0, n \geqslant 1,\right.
\end{gathered}
$$

and $B(A, N) \stackrel{s_{N}}{\longrightarrow} B(A, N)$ by

$$
s_{N}\left(a\left[a_{1}|\ldots| a_{n}\right] x\right)=\left[a\left|a_{1}\right| \ldots \mid a_{n}\right] x .
$$


Now consider the special case when $N=A / I$ for an ideal $I$ of $A$. Let $A / I \stackrel{\mathrm{nf}_{I}}{\longrightarrow} A$ be given by normal form (Remark 3.5) (with respect to some term ordering). One then has an SDR

$$
A / I \underset{\mathrm{nf}_{I}}{\stackrel{\sigma_{A / I}}{\rightleftarrows}}\left(B(A, A / I), s_{A / I}\right)
$$

and hence a free $A$-resolution of $A / I$ over $A$ with explicit contracting homotopy $s_{A / I}$. We call this the bar resolution of $A / I$ over $A$.

\section{Explicit Realizations of the Comparison Theorem}

It is well known that given any two resolutions $X \longrightarrow N$ and $Y \longrightarrow N$ of $N$ over $A$, there are chain maps $X \stackrel{f}{\longrightarrow} Y$ and $Y \stackrel{g}{\longrightarrow} X$ such that $f g$ is chain homotopic to $1_{Y}$ and $g f$ is chain homotopic to $1_{X}$, i.e. two sided data (2.3) exists. This is called the comparison theorem [23]. When $X$ is relatively free, and $Y$ possesses an explicit contracting homotopy $\psi$, there is a canonical such map $f$ given by an inductive formula [23, Chapter IV, §6].

\subsection{The Relatively Free Case}

When both $X$ and $Y$ are relatively free and there are explicit SDRs data

$$
N \underset{\epsilon_{X}}{\stackrel{\sigma_{X}}{\rightleftarrows}}\left(X, \psi_{X}\right)
$$

and

$$
N \underset{\epsilon_{Y}}{\stackrel{\sigma_{Y}}{\rightleftarrows}}\left(Y, \psi_{Y}\right)
$$

there are inductive formulae for canonical chain homotopy equivalences $f$ and $g$ as above as well as for the homotopies. The construction was used in $[\mathbf{6}]$ and is crucial for the work in $[\mathbf{1 6}],[\mathbf{1 9}],[\mathbf{2 0}],[\mathbf{1 8}]$, and [26]. Also see [23, Chapter IX]. Generally, one can only obtain two sided data (see 2.3). In some important situations however, an SDR (see 2.1) arises, as will be seen.

The $A$-linear chain maps $f$ and $g$ and $A$-linear chain homotopies $\phi_{X}$ and $\phi_{Y}$ referred to above are defined by induction. They are first defined on reduced elements and then extended $A$-linearly.

Take $f_{0}$ to be the $A$-linear extension of

$$
\left.\left(f_{0}\right)\right|_{\bar{X}_{0}}=\left.\left(\sigma_{Y} \epsilon_{X}\right)\right|_{\bar{X}_{0}}
$$

and $g_{0}$ to be the $A$-linear extension of

$$
\left.\left(g_{0}\right)\right|_{\bar{Y}_{0}}=\left.\left(\sigma_{X} \epsilon_{Y}\right)\right|_{\bar{Y}_{0}}
$$

Next take $f_{n}$ to be the $A$-linear extension of $\psi_{X} f_{n-1} d_{X}$ and $g_{n}$ the $A$-linear extension of $\psi_{Y} g_{n-1} d_{Y} . \phi_{X}$ and $\phi_{Y}$ are constructed similiarly. 


\subsubsection{The SDR Case}

We would like to know when the recursively defined maps above are maps in an SDR. A condition for $\nabla$ to be one-one was given in [25], viz. $d(\bar{X}) \cap \bar{X}=0$. This however is not enough to ensure that $f \nabla=1$ as the following example shows.

Example 4.1. Let $M=\left(m_{1}, m_{2}, m_{3}\right)$ where $m_{1}=x^{3} y^{2}, m_{2}=x^{3} y z$, and $m_{3}=$ $x y^{5} z$ and let $T$ be the Taylor resolution as in Example 3.3. We have

$$
\begin{gathered}
d u_{1}=m_{1}, d u_{2}=m_{2}, d u_{3}=m_{3}, \\
d u_{1} u_{2}=-z u_{1}+y u_{2}, d u_{1} u_{3}=-y^{3} z u_{1}+x^{2} u_{3},
\end{gathered}
$$

and

$$
d u_{2} u_{3}=-y^{4} u_{2}+x^{2} u_{3}, d u_{1} u_{2} u_{3}=y^{3} u_{1} u_{2}-u_{1} u_{3}+u_{2} u_{3} .
$$

Using the construction above, we have $T \stackrel{\nabla}{\longrightarrow} B(A, A / M)$ and $B(A, A / M) \stackrel{f}{\longrightarrow} T$ where $\nabla(a)=a[]$ and $f(a[])=a$ for $a \in A$ and

$$
\nabla u_{i}=s \nabla m_{i}=s\left(m_{i}[]\right)=\left[m_{i}\right] .
$$

Also,

$$
\nabla\left(u_{i} u_{j}\right)=s \nabla\left(\frac{m_{\{i, j\}}}{m_{j}} u_{j}-\frac{m_{\{i, j\}}}{m_{i}} u_{i}\right)=\left[\frac{m_{\{i, j\}}}{m_{j}} \mid m_{j}\right]-\left[\frac{m_{\{i, j\}}}{m_{i}} \mid m_{i}\right] .
$$

In particular,

$$
\nabla\left[u_{2} \mid u_{3}\right]=\left[x^{2} \mid m_{3}\right]-\left[y^{4} \mid m_{2}\right] .
$$

But consider $f$ now. We have

$$
f \nabla\left[u_{i}\right]=\psi\left(f \partial\left(\left[m_{i}\right]\right)=\psi\left(f\left(m_{i}[]\right)\right)\right.
$$

since [ $]\left(m_{i}+M\right)=0$ in $B(A, A / M)$ and so

$$
f \nabla\left[u_{i}\right]=\psi\left(m_{i}\right)=u_{i}
$$

for all $i$. However, it follows that

$$
f\left(\left[x^{2} \mid m_{3}\right]\right)=\psi f\left(x^{2}\left[m_{3}\right]-\left[x^{2} m_{3}\right]\right)=\psi\left(x^{2} \psi\left(m_{3}\right)-\psi\left(x^{2} m_{3}\right)\right)=\psi\left(x^{2} \psi\left(m_{3}\right)\right)
$$

since $\psi^{2}=0$. Similarly, it follows that

$$
f\left(\left[y^{4} \mid m_{2}\right]\right)=\psi\left(y^{4} \psi\left(m_{2}\right)\right)
$$

and so

$$
f\left(\left[x^{2} \mid m_{3}\right]\right)=\psi\left(x^{2} u_{3}-y^{4} u_{2}\right)=-y^{3} u_{1} u_{2}+u_{1} u_{3} \neq u_{1} u_{2} .
$$

Note that this example satisfies the condition from [25] stated above. The given maps do however form two sided data.

There is a condition however that will give rise to SDR data.

Lemma 4.2. Suppose that $X$ is a relatively free resolution as above and that the contracting homotopy $\psi_{X}$ satisfies $\psi(\bar{X})=0$ and $d(\bar{X}) \cap \bar{X}=0$ and the homotopy 
$\psi_{Y}$ satisfies $\psi_{Y}(Y) \subseteq \bar{Y}$, then the constructions above give an SDR

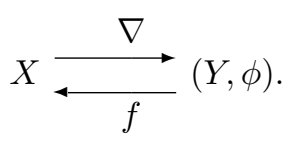

Proof. The proof is by induction. We have $f_{0} \nabla_{0}=1$ by construction and for $n>0$, on reduced elements,

$$
\begin{aligned}
f_{n} \nabla_{n}=f_{n} \psi_{Y} \nabla_{n-1} d_{X} & =\psi_{X} f_{n-1} d_{Y} \psi_{Y} \nabla_{n-1} d_{X} \\
& =\psi_{X} f_{n-1}\left(1-\psi_{Y} d_{Y}\right) \nabla_{n-1} d_{X},
\end{aligned}
$$

where the first equality comes from the hypothesis on $\psi_{Y}$. But

$$
\begin{aligned}
\psi_{X} f_{n-1} \psi_{Y} d_{Y} \nabla_{n-1} d_{X} & =\psi_{X} f_{n-1} \psi_{Y} \nabla_{n-1} d_{X} d_{X} \\
& =0
\end{aligned}
$$

and by induction and the hypothesis on $\psi_{X}$,

$$
\begin{aligned}
\psi_{X} f_{n-1} \nabla_{n-1} d_{X} & =\psi_{X} d_{X} \\
& =1 .
\end{aligned}
$$

Thus from Lemma 3.8, we have the

Corollary 4.3. Let $M$ be a monomial ideal and $L$ the Lyubeznik resolution of $A / M$ over $A$. We have an $S D R$

$$
L \underset{f}{\stackrel{\nabla}{\longrightarrow}}(B(A, A / M), \phi) .
$$

\subsection{Explicit Maps for Splitting the Lyubeznik Resolution off of the Bar Construction in Low Degree}

Examples of the maps from Corollary 4.3 were given in Example 4.1. We will give some more explicit formulae here. Let $M$ and $L$ be as in the last section. Note that we are assuming that we have chosen some term ordering on $A$ so that we have the SDR

$$
A / M \underset{\mathrm{nf}_{\mathrm{M}}}{\stackrel{\sigma}{\longrightarrow}}(B(A, A / M), \phi),
$$

where $\mathrm{nf}_{\mathrm{M}}$ denotes the normal form map with respect to $M$ and the term ordering on $A$.

In general, the map $\nabla$ is easy. In degree zero,

$$
\nabla(a)=a[], a \in A
$$

and in degree greater than zero,

$$
\nabla\left(u_{i}\right)=s \nabla d u_{i}=\left[m_{i}\right],
$$




$$
\begin{gathered}
\nabla\left(u_{i} u_{j}\right)=\left[\frac{m_{i, j}}{m_{j}} \mid m_{j}\right]-\left[\frac{m_{i, j}}{m_{i}} \mid m_{i}\right], \\
\nabla\left(u_{i} u_{j} u_{k}\right)=\left[\frac{m_{i, j, k}}{m_{k}} \mid \frac{m_{i, j}}{m_{j}} m_{j}\right]-\left[\frac{m_{i, j, k}}{m_{k}} \mid \frac{m_{i, j}}{m_{i}} m_{i}\right]-\left[\frac{m_{i, j, k}}{m_{k}} \mid \frac{m_{i, k}}{m_{k}} m_{k}\right]+ \\
{\left[\frac{m_{i, j, k}}{m_{k}} \mid \frac{m_{i, k}}{m_{i}} m_{i}\right]+\left[\frac{m_{i, j, k}}{m_{k}} \mid \frac{m_{j, k}}{m_{k}} m_{k}\right]-\left[\frac{m_{i, j, k}}{m_{k}} \mid \frac{m_{j, k}}{m_{j}} m_{j}\right],}
\end{gathered}
$$

etc.

The map $f$ is a bit more involved due to the fact that $B_{0}(A, A / M)=A \otimes_{k} A / M$. Using the initial formula for $f$ in Section 4.1, we have

$$
f_{0}([](b+M))=\operatorname{nf}_{\mathrm{M}}(b)+M .
$$

So that

$$
f_{1}\left(\left[a_{1}\right](b+M)\right)=\psi f\left(a_{1}[] b-[]\left(a_{1} b+M\right)\right)=\psi\left(a_{1} \operatorname{nf}_{\mathrm{M}}(b)-\operatorname{nf}_{\mathrm{M}}\left(a_{1} b\right)\right) .
$$

Using the fact that $\psi^{2}=0$, we can also see that

$$
f_{2}\left(\left[a_{1} \mid a_{2}\right](b+M)\right)=\psi\left(a_{1} \psi\left(a_{2} \operatorname{nf}_{\mathrm{M}}(b)-\operatorname{nf}_{\mathrm{M}}\left(a_{2} b\right)\right),\right.
$$

etc.

The map $\phi$ is just as straightforward:

$$
\begin{gathered}
\phi_{0}([](b+M))=\left[\operatorname{nf}_{\mathrm{M}}(b)\right] . \\
\phi_{1}\left(\left[a_{1}\right](b+M)\right)=s \nabla \psi\left(a_{1} \operatorname{nf}_{\mathrm{M}}(b)-\operatorname{nf}_{\mathrm{M}}\left(a_{1} b\right)\right)-\left[a_{1} \mid \operatorname{nf}_{\mathrm{M}}(b)\right],
\end{gathered}
$$

etc. Further degrees are left as an exercise to the interested reader.

\section{Main Result}

\subsection{Perturbing Monomial Resolutions}

Here is the main result.

Theorem 5.1. Let $I=\left(f_{1}, \ldots, f_{r}\right)$ be an ideal in $A$ and choose a monomial order. Let $G=\left\{g_{1}, \ldots, g_{s}\right\}$ be a Gröbner basis for I with respect to the given order. Let $M=\mathrm{LT}(G)=\left(m_{1}, \ldots, m_{s}\right)$ be the ideal generated by the leading terms of $G$. Let $L$ be the Lyubeznik resolution of $A / M$ over $A$. There is an explicit perturbation $p$ of the differential $d$ in $L$ so that $(L, d+p)$ is a resolution of $A / I$ over $A$.

Proof. Consider the SDR

$$
L \underset{f}{\stackrel{\nabla}{\rightleftarrows}}(B(A, A / M), \phi)
$$

given by the corollary above. Using the vector space isomorphism

$$
A / M \approx A / I
$$

[8], [3], we obtain a vector space isomorphism of $B(A, A / M)$ and $B(A, A / I)$. Using this isomorphism, $B(A, A / M)$ supports two differentials, the one for $A / I$ and the 
one for $A / M$. Let $t$ be the difference of these differentials. A transference problem [1] has just been set up and the basic perturbation lemma gives a formal solution. If the formal solution converges, we obtain a new differential $d_{\infty}$, where $d_{\infty}=d+p$ and a new SDR

$$
\left(L, d_{\infty}\right) \underset{f_{\infty}}{\stackrel{\nabla_{\infty}}{\rightleftarrows}}\left(B(A, A / I), \phi_{\infty}\right)
$$

as in Section 2.2.

We have

$$
t\left(\left[a_{1}|\ldots| a_{n}\right](b+M)\right)=(-1)^{n}\left[a_{1}|\ldots| a_{n-1}\right]\left(\operatorname{nf}_{\mathrm{G}}\left(a_{n} b\right)-\operatorname{nf}_{\mathrm{M}}\left(a_{n} b\right)+M\right) .
$$

For convergence, it suffices to see that $t \phi$ is nilpotent in each finite degree. This can be seen by examining what is going on in low degrees first. In degree zero, we have

$$
t \phi([](b+M))=-[]\left(\operatorname{nf}_{\mathrm{M}}(q)+M\right)
$$

where $q=\operatorname{nf}_{\mathrm{G}}(b)-\mathrm{nf}_{\mathrm{M}}(b)$. Continuing, we thus have

$$
t \phi t \phi([](b+M))=[]\left(\operatorname{nf}_{\mathrm{M}}\left(q^{\prime}\right)+M\right)
$$

where $q^{\prime}=\operatorname{nf}_{\mathrm{G}}\left(\operatorname{nf}_{\mathrm{M}}(q)\right)-\mathrm{nf}_{\mathrm{M}}\left(\mathrm{nf}_{\mathrm{M}}(q)\right)$ But clearly, $\mathrm{nf}_{\mathrm{Z}}\left(\mathrm{nf}_{\mathrm{M}}(b)\right)=\mathrm{nf}_{\mathrm{M}}(b)$ for all $b$ where $Z$ is either $G$ or $M$. Thus, in degree zero, $t \phi$ is nilpotent of order at most two. In degree one, things are a bit more complicated. From our formula for $\phi$ in the last section, we have

$$
\phi([a](b+M))=\sum\left[s_{i} \mid m_{i}\right]+\left[a \mid \operatorname{nf}_{\mathrm{M}}(b)\right]
$$

where

$$
\psi\left(a \operatorname{nf}_{\mathrm{M}}(b)-\mathrm{nf}_{\mathrm{M}}(a b)\right)=\sum_{i} s_{i} u_{i} .
$$

Note that we actually have $\psi\left(a \operatorname{nf}_{\mathrm{M}}(b)-\mathrm{nf}_{\mathrm{M}}(a b)\right)=\psi\left(a \mathrm{nf}_{\mathrm{M}}(b)\right)$ since no $m_{k}$ can divide the normal form $\mathrm{nf}_{\mathrm{M}}(a b)$ and therefore $\psi$ vanishes on it. In any case, we thus have

$$
t \phi([a](b+M))=-\sum\left[s_{i}\right]\left(\operatorname{nf}_{\mathrm{G}}\left(m_{i}\right)+M\right)
$$

since $\operatorname{nf}_{\mathrm{G}}\left(\mathrm{nf}_{\mathrm{M}}(b)\right)-\mathrm{nf}_{\mathrm{M}}\left(\mathrm{nf}_{\mathrm{M}}(b)\right)=0$ and $\mathrm{nf}_{\mathrm{M}}\left(m_{i}\right)=0$ for all $i$. But note that

$$
\phi\left(\left[s_{i}\right](q+M)\right)=-s\left(\nabla\left(\psi\left(s_{i} \operatorname{nf}_{\mathrm{M}}(q)\right)\right)\right)+\left[s_{i} \mid \operatorname{nf}_{\mathrm{M}}(q)\right]
$$

where $q=\operatorname{nf}_{\mathrm{G}}\left(m_{i}\right)$ Now note that strictly fewer $m_{j}$ can divide $s_{i} \operatorname{nf}_{\mathrm{M}}(q)$ than could divide the original $a \mathrm{nf}_{\mathrm{M}}(b)$. Thus repeated applications of $t \phi$ will eventually vanish because of the action of $\psi$. The situation in higher degrees is similar but slightly more complicated and is left to the interested reader.

Remark 5.2. In fact, a contracting homotopy for the resolution $\left(L, d_{\infty}\right) \rightarrow A / I \rightarrow$ 0 over $A$ is explicitly given by

$$
\psi_{\infty}=f_{\infty} s \nabla_{\infty}
$$

where $s$ is the contracting homotopy on the bar resolution. 


\section{Examples of the Main Algorithm}

\subsection{Example I}

Consider the polynomial ring $A=k[x, y, z]$ over a field $k$ and the ideal $I$ generated by

$$
\begin{aligned}
& f_{1}=x^{3} y^{2}-x^{2} y z^{2}+x y z \\
& f_{2}=x y^{5} z-x y z .
\end{aligned}
$$

Using the lexicographic order on monomials, we have computed a Gröbner basis $G=\left\{g_{1}, g_{2}, g_{3}\right\}$ where

$$
\begin{aligned}
& g_{1}=x^{3} y^{2}-x^{2} y z^{2}+x y z \\
& g_{2}=x^{3} y z-x^{2} y^{4} z^{3}+x y^{4} z^{2} \\
& g_{3}=x y^{5} z-x y z .
\end{aligned}
$$

Now consider the monomial ideal $M=\left(x^{3} y^{2}, x^{3} y z, x y^{5} z\right)$ generated by the leading terms of the polynomials in $G$. Let $m_{1}=x^{3} y^{2}, m_{2}=x^{3} y z$, and $m_{3}=x y^{5} z$. As is well-known, $\left\{m_{1}, m_{2}, m_{3}\right\}$ is a Gröbner basis of $M$. Note that this monomial ideal has been discussed in Example 4.1.

The Taylor differential is given by

$$
\begin{aligned}
d u_{i} & =m_{i}, i=1,2,3 \\
d u_{1} u_{2} & =-z u_{1}+y u_{2} \\
d u_{1} u_{3} & =-y^{3} z u_{1}+x^{2} u_{3} \\
d u_{2} u_{3} & =-y^{4} u_{2}+x^{2} u_{3} \\
d u_{1} u_{2} u_{3} & =y^{3} u_{1} u_{2}-u_{1} u_{3}+u_{2} u_{3} .
\end{aligned}
$$

As noted in Example 4.1, since $\psi$ does not vanish on all reduced elements, we cannot use Lemma 4.2 to get an SDR with the bar resolution. Note however that Example 3.3 shows that we could reorder the monomials and then Lemma 4.2 would apply. However, since the Lyubeznik Resolution is smaller in this case, we will proceed with it instead.

6.1.1. Splitting the Lyubeznik Resolution Off from the Bar Resolution It is not hard to calculate that $L=\left\langle u_{1}, u_{2}, u_{3}, u_{1} u_{2}, u_{1} u_{3}\right\rangle$ in this case. The differential is thus given by the first five maps above. Notice that the reduced complex has zero differential and so $L$ is actually the minimal resolution. Using the constructions from Section 4.1, it is not hard to see that we have

$$
\begin{aligned}
\nabla u_{i} & =\left[m_{i}\right] \\
\nabla u_{1} u_{2} & =\left[y \mid m_{2}\right]-\left[z \mid m_{1}\right] \\
\nabla u_{1} u_{3} & =\left[x^{2} \mid m_{3}\right]-\left[y^{3} z \mid m_{1}\right] .
\end{aligned}
$$

Continuing, we have the following formulae for the projection $f$. On reduced elements

$$
\begin{aligned}
f([] b) & =\operatorname{nf}_{\mathrm{M}}(b)[] \\
f\left(\left[a_{1}\right] b\right) & =\psi\left(a_{1} \operatorname{nf}_{\mathrm{M}}(b)\right) .
\end{aligned}
$$


This is all that is needed to calculate the perturbation based on the initiator

$$
t\left(\left[a_{1}|\ldots| t_{n}\right](b+M)\right)=(-1)^{n}\left[a_{1}|\ldots| a_{n-1}\right]\left(\operatorname{nf}_{\mathrm{G}}\left(a_{n} b\right)-\mathrm{nf}_{\mathrm{M}}\left(a_{n} g\right)+M\right) .
$$

We obtain the following resolution of $A / I$ over $A$ in this manner.

$$
\begin{aligned}
d\left(u_{i}\right) & =g_{i} \text { for } i=1,2,3, \\
d\left(u_{1} u_{2}\right) & =-z u_{1}+y u_{2}+\left(x z^{2}-z\right) u_{3} \\
d\left(u_{1} u_{3}\right) & =-y^{3} z u_{1}+u_{2}+x^{2} u_{3} .
\end{aligned}
$$

It follows that

$$
\operatorname{Tor}^{A}(k, A / I)=\left\langle u_{1}, u_{1} u_{2}\right\rangle .
$$

Furthermore, using $\nabla_{\infty}$ we can calculate representatives for these homology classes. We have

$$
\begin{aligned}
\nabla_{\infty}\left(u_{1}\right) & =\left[x^{3} y^{2}\right]-\left[x^{2} y z^{2}-x y z\right] \\
\nabla_{\infty}\left(u_{1} u_{2}\right) & =\left[x z^{2}-z \mid x y^{5} z\right]+\left[y \mid x^{3} y z\right]+\left[z \mid x^{2} y z^{2}-x y z\right] \\
& -\left[z \mid x^{3} y^{2}\right]-\left[y \mid x^{2} y^{4} z^{3}-x y^{4} z^{2}\right]+\left[-x z^{2}+z \mid x y z\right]
\end{aligned}
$$

Remark 6.1. Note that the term ordering used makes a considerable difference. Using the same two polynomials $f_{1}$ and $f_{2}$ above, but the total order refined by reverse lex instead of pure lex, we obtain a Gröbner basis with six elements. Also, in this case the Lyubeznik resolution is the same as the Taylor resolution. Thus, in this case, in terms of size, the pure lex order is a better choice.

\subsection{Example II}

In this example, the Taylor resolution will not split off of the bar construction. We will however use the Lyubznek resolution to actually compute the minimal resolution of the monomial ideal and split that off of the bar construction and then proceed as before.

Let $f_{1}=x y+x z+x w, f_{2}=y z+y w$, and $f_{3}=z w$ and let $I=\left(f_{1}, f_{2}, f_{3}\right)$. Using the pure lex monomial order, a Gröbner basis $G=\left\{g_{1}, \ldots, g_{6}\right\}$ is given by $g_{1}=x y+x z+x w, g_{2}=x z^{2}+x w^{2}, g_{3}=x w^{3}, g_{4}=y z+y w, g_{5}=y w^{2}$, and $g_{6}=z w$. The ideal $M$ generated by the leading terms thus has Gröbner basis $\left\{m_{1}, \ldots, m_{6}\right\}$ where $m_{1}=x y, m_{2}=x z^{2}, m_{3}=x w^{3}, m_{4}=y z, m_{5}=y w^{2}$, and $m_{6}=z w$.

6.2.1. The Lyubeznik Resolution

Direct computation gives that $L$ is generated by $u_{i}$ for $i=1, \ldots, 6, u_{i} u_{j}$ for

$$
(i, j) \in\{(1,2),(1,3),(1,4),(1,5),(1,6),(2,3),(2,6),(3,6),(4,5),(4,6)\},
$$

$u_{i} u_{j} u_{k}$ for

$$
(i, j, k) \in\{(1,2,3),(1,2,6),(1,3,6),(1,4,5),(2,3,6)\},
$$

and $u_{1} u_{2} u_{3} u_{6}$. 
The reduced differential in this case is non-zero only in the following cases:

$$
\begin{aligned}
\bar{d}\left(u_{1} u_{4} u_{6}\right) & =-u_{1} u_{6} \\
\bar{d}\left(u_{2} u_{3} u_{6}\right) & =u_{2} u_{3} \\
\bar{d}\left(u_{1} u_{2} u_{3} u_{6}\right) & =-u_{1} u_{2} u_{3} .
\end{aligned}
$$

6.2.2. The Minimal Resolution

It is straightforward to see that a homology decomposition (Section 7 ) is given by

$$
\bar{L}=K \oplus B \oplus H
$$

where

$$
\begin{aligned}
K & =\left\langle u_{1} u_{4} u_{6}, u_{2} u_{3} u_{6}, u_{1} u_{2} u_{3}\right\rangle \\
B & =\left\langle u_{1} u_{6}, u_{2} u_{3}, u_{1} u_{2} u_{3}\right\rangle .
\end{aligned}
$$

Thus, the (A-linear) homotopy $\bar{\phi}$ is non-zero only in the following cases:

$$
\begin{aligned}
\bar{\phi}\left(u_{1} u_{6}\right) & =u_{1} u_{4} u_{6} \\
\bar{\phi}\left(u_{2} u_{3}\right) & =-u_{2} u_{3} \\
\bar{\phi}\left(u_{1} u_{2} u_{3}\right) & =u_{1} u_{2} u_{3} u_{6} .
\end{aligned}
$$

This give us an SDR

$$
H \underset{\bar{f}}{\stackrel{\bar{\nabla}}{\rightleftarrows}}((\bar{L}, \bar{d}), \bar{\phi})
$$

where $\bar{\nabla}$ is the inclusion. Consider the SDR

$$
A \otimes H \underset{f}{\stackrel{\nabla}{\rightleftarrows}}((L, 1 \otimes \bar{d}), \phi)
$$

where $\nabla=1_{A} \otimes \bar{\nabla}, f=1_{A} \otimes \bar{f}$, and $\phi=1_{A} \otimes \bar{\phi}$. Using the initiator $t=d-1_{A} \otimes \bar{d}$ on $L$, we have a transference problem.

Proposition 6.2. The formal solution to the transference problem above converges and we have thus derived the minimal resolution given by the following A-linear differential $\delta=d_{\infty}$ on $A \otimes H$. We have $\delta=d$ except for the cases:

$$
\begin{aligned}
& \delta\left(u_{1} u_{2} u_{6}\right)=d\left(u_{1} u_{2} u_{6}\right)+z u_{1} u_{6}-z u_{1} u_{4}-x z u_{4} u_{6} \\
& \delta\left(u_{1} u_{3} u_{6}\right)=d\left(u_{1} u_{3} u_{6}\right)+w u_{1} u_{6}-w^{3} u_{1} u_{4}-x w^{2} u_{4} u_{6} .
\end{aligned}
$$

6.2.3. Splitting the Minimal Resolution Off From the Bar Resolution We have the minimal resolution $(A \otimes H, \delta)$ above and an explicit contracting homotopy $\psi_{\infty}=f_{\infty} \psi \nabla_{\infty}$ where $\psi$ is the contracting homotopy for the Lyubeznik (and Taylor) resolution. An explicit calculation shows that $\psi_{\infty}$ vanishes on reduced 
elements and hence we have an SDR

$$
(A \otimes H, \delta) \underset{p}{\stackrel{i}{\rightleftarrows}}(B(A, A / M), h)
$$

by Lemma 4.2. The perturbation converges and we have

Proposition 6.3. The formal solution to the transference problem above converges and we have an $A$-free resolution of $A / I$ given by

$$
\begin{aligned}
\delta_{\infty}\left(u_{i}\right) & =g_{i} \text { for } i=1, \ldots, 6 \\
\delta_{\infty}\left(u_{1} u_{2}\right) & =\left(-z^{2}-w^{2}\right) u_{1}+(y+z+w) u_{2} \\
\delta_{\infty}\left(u_{1} u_{3}\right) & =-w^{3} u_{1}+(y+z+w) u_{3} \\
\delta_{\infty}\left(u_{1} u_{4}\right) & =(-z-w) u_{1}+u_{2}+x u_{4}+2 x u_{6} \\
\delta_{\infty}\left(u_{1} u_{5}\right) & =-w^{2} u_{1}+u_{3}+x u_{6}+x w u_{6} \\
\delta_{\infty}\left(u_{2} u_{6}\right) & =-w u_{2}+u_{3}+x z u_{6} \\
\delta_{\infty}\left(u_{3} u_{6}\right) & =-z u_{3}+x w^{2} u_{6} \\
\delta_{\infty}\left(u_{4} u_{5}\right) & =-w^{2} u_{4}+(z+w) u_{5} \\
\delta_{\infty}\left(u_{4} u_{6}\right) & =-w u_{4}+u_{5}+y u_{6} \\
\delta_{\infty}\left(u_{1} u_{2} u_{6}\right) & =w u_{1} u_{2}-u_{1} u_{3}-z w u_{1} u_{4}+z u_{1} u_{5}+(y+w) u_{2} u_{6}-x z u_{4} u_{6} \\
\delta_{\infty}\left(u_{1} u_{3} u_{6}\right) & =z u_{1} u_{3}-w^{3} u_{1} u_{4}+w^{2} u_{1} u_{5}-w^{2} u_{2} u_{6}+(y+z+w) u_{3} u_{6}-x w^{2} u_{4} u_{6} \\
\delta_{\infty}\left(u_{1} u_{4} u_{5}\right) & =w^{2} u_{1} u_{4}+(-z-w) u_{1} u_{5}+w u_{2} u_{6}-u_{3} u_{6}+x u_{4} u_{5} .
\end{aligned}
$$

\section{The SDR Given by a Homology Decomposition}

For the reader's convenience, the well-known [13] homology decomposition will be reviewed in this section. We cast it into the context of SDRs. We continue to work over the field $k$, but all of what we say generalizes to torsion-free modules over a principal ideal domain.

\subsection{Homology}

Let $f$ and $g$ be linear transformations such that

$$
g: R^{c} \rightarrow R^{b}, f: R^{b} \rightarrow R^{a}
$$

and $f g=0$. Let $F$ and $G$ be the matrices of $f$ and $g$ with respect to the standard bases. We will generally write $M_{n \times m}$ to denote that $M$ has size $n \times m$, thus $F=F_{a \times b}$ and $G=G_{b \times c}$.

Let $D=P F Q$ be the Smith normal form $([\mathbf{2 4}])$ of $F$ so that $P=P_{a \times a}$ and $Q=Q_{b \times b}$ are invertible and $D=D_{a \times b}$ diagonal matrix of the form

$$
D=\left[\begin{array}{ll}
I_{r \times r} & O_{r \times(b-r)} \\
O_{(a-r) \times r} & O_{(a-r) \times(b-r)}
\end{array}\right]
$$

$I$ denotes the identity matrix, $O$ denotes the matrix with all entries zero, and 
$r=\operatorname{rank}_{R}(F)$. Since $D Q^{-1} G=P F G=O$, it follows that

$$
Q^{-1} G=\left[\begin{array}{l}
O_{r \times c} \\
\widetilde{G}_{(b-r) \times c}
\end{array}\right] .
$$

Now consider the Smith normal form $E=\tilde{T} \tilde{G} S$, where

$$
E=\left[\begin{array}{ll}
I_{r^{\prime} \times r^{\prime}} & O_{r^{\prime} \times\left(c-r^{\prime}\right)} \\
O_{\left(b-r-r^{\prime}\right) \times r^{\prime}} & O_{\left(b-r-r^{\prime}\right) \times\left(c-r^{\prime}\right)}
\end{array}\right] .
$$

Set

$$
T_{b \times b}=\left[\begin{array}{ll}
I_{r \times r} & O_{r \times(b-r)} \\
O_{(b-r) \times r} & \widetilde{T}_{(b-r) \times(b-r)}
\end{array}\right] .
$$

It follows that

$$
T Q^{-1} G S=\left[\begin{array}{l}
O_{r \times c} \\
E
\end{array}\right]=\left[\begin{array}{ll}
O_{r \times r^{\prime}} & O_{r \times\left(c-r^{\prime}\right)} \\
I_{r^{\prime} \times r^{\prime}} & O_{r^{\prime} \times\left(c-r^{\prime}\right)} \\
O_{\left(b-r-r^{\prime}\right) \times r^{\prime}} & O_{\left(b-r-r^{\prime}\right) \times\left(c-r^{\prime}\right)}
\end{array}\right] .
$$

Thus, letting $\left\{e_{i}\right\}$ denote the standard bases, we have

$$
\operatorname{PFQT}^{-1}\left(e_{i}\right)= \begin{cases}e_{i}, & 1 \leqslant i \leqslant r \\ 0, & r+1 \leqslant i \leqslant b\end{cases}
$$

and

$$
T Q^{-1} G S\left(e_{j}\right)=\left\{\begin{array}{ll}
e_{j+r}, & 1 \leqslant j \leqslant r^{\prime} \\
0, & r^{\prime}+1 \leqslant j \leqslant c
\end{array} .\right.
$$

We thus have bases $\alpha_{i}[1]=S\left(e_{i}\right), 1 \leqslant i \leqslant c, \alpha_{i}[0]=Q T^{-1}\left(e_{i}\right), 1 \leqslant i \leqslant b$, and $\alpha_{i}[-1]=P^{-1}\left(e_{i}\right), 1 \leqslant i \leqslant a$ such that

$$
f\left(\alpha_{i}[0]\right)= \begin{cases}\alpha_{i}[-1], & 1 \leqslant i \leqslant r \\ 0, & r+1 \leqslant i \leqslant b\end{cases}
$$

and

$$
g\left(\alpha_{j}[1]\right)=\left\{\begin{array}{ll}
\alpha_{r+j}[0], & 1 \leqslant j \leqslant r^{\prime} \\
0, & r^{\prime}+1 \leqslant j \leqslant c
\end{array} .\right.
$$

It is immediate that

$$
\operatorname{ker}(f)=\left\langle\alpha_{r+1}[0], \ldots, \alpha_{b}[0]\right\rangle
$$

and

$$
\operatorname{im}(g)=\left\langle\alpha_{r+1}[0], \ldots, \alpha_{r+r^{\prime}}[0]\right\rangle,
$$

so that we have the well-known homology decomposition [13]

$$
R^{b}=\left\langle\alpha_{1}[0], \ldots, \alpha_{r}[0]\right\rangle \oplus\left\langle\alpha_{r+1}[0], \ldots, \alpha_{r+r^{\prime}}[0]\right\rangle \oplus\left\langle\alpha_{r+r^{\prime}+1}[0], \ldots, \alpha_{b}[0]\right\rangle .
$$

\subsection{Homology SDR}

Let $(X, d)$ by a chain complex of finite type (i.e. $H_{n}(X)$ is finite dimensional for all $n$ ). We want to give a special presentation which can be used to calculate minimal resolutions of the type we have written about in the sections above. Let $\left\{e_{s}[i] \mid 1 \leqslant\right.$ 
$\left.s \leqslant n_{i}\right\}$ be a basis for $X_{i}$. In the notation of the preceding Section, we have bases $\left\{\alpha_{s}(0,0)=R^{-1} e_{s}[0]\right\}$ for $X_{0}$ and $\left\{\alpha_{s}(0,1)=S e_{s}[1]\right\}$ for $X_{1}$. Furthermore, we have

$$
d_{1}\left(\alpha_{s}(0,1)\right)=\left\{\begin{array}{ll}
\alpha_{s}(0,0), & 1 \leqslant j \leqslant r_{1} \\
0, & r_{1}+1 \leqslant j \leqslant n_{1}
\end{array} .\right.
$$

More generally, we have $P_{i}$ of order $n_{i-1} \times n_{i-1}, Q_{i}$ of order $n_{i} \times n_{i}, \widetilde{T}_{i}$ of order $\left(n_{i}-r_{i}\right) \times\left(n_{i}-r_{i}\right), T_{i}$ of order $n_{i} \times n_{i}$ and $S_{i}$ of order $n_{i+1} \times n_{i+1}$ giving bases $\left\{\alpha_{s}(i, 0)=Q_{i} T_{i}^{-1} e_{s}[i]\right\}$ for $X_{i},\left\{\alpha_{s}(i,-1)=P_{i}^{-1} e_{s}[i-1]\right\}$ for $X_{i-1}$, and $\left\{\alpha_{s}(i, 1)=\right.$ $\left.S_{i} e_{s}[i+1]\right\}$ for $X_{i+1}$. We define

$$
\begin{gathered}
K_{i}=\left\langle\alpha_{1}(i, 0), \ldots, \alpha_{r_{i}}(i, 0)\right\rangle \\
B_{i}=\left\langle\alpha_{r_{i}+1}(i, 0), \ldots, \alpha_{r_{i}+r_{i+1}}(i, 0)\right\rangle
\end{gathered}
$$

and

$$
H_{i}=\left\langle\alpha_{r_{i} r_{i+1}+1}(i, 0), \ldots, \alpha_{n_{i}}(i, 0)\right\rangle
$$

We thus have

$$
X_{i}=K_{i} \oplus B_{i} \oplus H_{i}
$$

with

$$
d_{i}\left(\alpha_{s}(i, 0)\right)= \begin{cases}\alpha_{s}(i,-1), & 1 \leqslant j \leqslant r_{i} \\ 0, & r_{i}+1 \leqslant j \leqslant n_{i}\end{cases}
$$

and

$$
d_{i+1}\left(\alpha_{r_{i}+s}(i, 1)\right)= \begin{cases}\alpha_{s}(i,-1), & 1 \leqslant j \leqslant r_{i+1} \\ 0, & r_{i+1}+1 \leqslant j \leqslant n_{i+1}\end{cases}
$$

Note that we have

$$
\left\langle\alpha_{r_{i}+1}(i, 0), \ldots, \alpha_{r_{i}+r_{i+1}}(i, 0)\right\rangle=\left\langle\alpha_{1}(i+1,-1), \ldots, \alpha_{r_{i+1}}(i+1,-1)\right\rangle .
$$

By equation (7.1), we can define a map

$$
\widetilde{\phi}_{i}: B_{i} \rightarrow K_{i+1}
$$

by

$$
\widetilde{\phi}_{i}\left(\alpha_{r_{i}+s}(i, 0)\right)=\alpha_{s}(i,-1)
$$

and we can extend this to a map $\phi$ of all of $X_{i}$ by taking $\phi_{i}$ to be zero on $K_{i}$ and $H_{i}$. It is left as an easy exercise to the reader to see that, in fact,

Theorem 7.1. The degree 1 map $\phi$ defined above is a splitting homotopy of $X$ and we have an $S D R$

$$
H \underset{f}{\stackrel{\nabla}{\longleftarrow}}(X, \phi) .
$$




\section{References}

[1] Donald W. Barnes and Larry A. Lambe. A fixed point approach to homological perturbation theory. Proc. Amer. Math. Soc., 112(3):881-892, 1991.

[2] Donald W. Barnes and Larry A. Lambe. Correction to "a fixed point approach to homological perturbation theory". Proc. Amer. Math. Soc., 129(3):941941, 2001.

[3] T. Becker, Kredel H., and V. Weispfennig. Gröbner Bases, A Computational Approach to Commutative Algebra. Graduate Texts in Math. Springer-Verlag, Berlin, NY, Heidelberg, 1993.

[4] R. Brown. The twisted Eilenberg-Zilber theorem. In Simposio di Topologia (Messina, 1964), pages 33-37. Edizioni Oderisi, Gubbio, 1965.

[5] Henri Cartan and Samuel Eilenberg. Homological algebra. Princeton University Press, Princeton, N. J., 1956.

[6] Henri Cartan, J. C. Moore, R. Thom, and J. P. Serre. Algèbras d'Eilenberg-MacLane et homotopie. 2ième ed., revue et corrigée. Secrétariat mathématique (Hektograph), Paris, 1956.

[7] Ralf Fröberg. Some complex constructions with applications to Poincaré series. In Séminaire d'Algèbre Paul Dubreil 31ème année (Paris, 1977-1978), pages 272-284. Springer, Berlin, 1979.

[8] Ralf Fröberg. An introduction to Gröbner bases. John Wiley \& Sons Ltd., Chichester, 1997.

[9] R.L. Graham, D.E. Knuth, and O. Patashnik. Concrete Mathematics. Addison Wesley, 1989.

[10] V. K. A. M. Gugenheim. On the chain-complex of a fibration. Illinois J. Math., 16:398-414, 1972.

[11] V. K. A. M. Gugenheim and L. A. Lambe. Perturbation theory in differential homological algebra. I. Illinois J. Math., 33(4):566-582, 1989.

[12] V. K. A. M. Gugenheim, L. A. Lambe, and J. D. Stasheff. Perturbation theory in differential homological algebra. II. Illinois J. Math., 35(3):357-373, 1991. 
[13] P. J. Hilton and S. Wylie. Homology theory: An introduction to algebraic topology. Cambridge University Press, New York, 1960.

[14] Johannes Huebschmann. The homotopy type of $F \psi^{q}$. The complex and symplectic cases. In Applications of algebraic K-theory to algebraic geometry and number theory, Part I, II (Boulder, Colo., 1983), pages 487-518. Amer. Math. Soc., Providence, R.I., 1986.

[15] Johannes Huebschmann and Tornike Kadeishvili. Small models for chain algebras. Math. Z., 207(2):245-280, 1991.

[16] Larry Lambe. Next generation computer algebra systems AXIOM and the scratchpad concept: applications to research in algebra. In Analysis, algebra, and computers in mathematical research (Luleå, 1992), volume 156 of Lecture Notes in Pure and Appl. Math., pages 201-222. Dekker, New York, 1994.

[17] Larry Lambe and Jim Stasheff. Applications of perturbation theory to iterated fibrations. Manuscripta Math., 58(3):363-376, 1987.

[18] Larry A. Lambe. Resolutions via homological perturbation. J. Pure Appl. Algebra, 12:71-87, 1991.

[19] Larry A. Lambe. Homological perturbation theory, Hochschild homology, and formal groups. In Deformation theory and quantum groups with applications to mathematical physics (Amherst, MA, 1990), volume 134 of Contemp. Math., pages 183-218. Amer. Math. Soc., Providence, RI, 1992.

[20] Larry A. Lambe. Resolutions which split off of the bar construction. J. Pure Appl. Algebra, 84(3):311-329, 1993.

[21] Larry A. Lambe. Entry on Homological Perturbation Theory in the Encyclopaedia of Mathematics. Supplement II. Kluwer Academic Press, 2000.

[22] Gennady Lyubeznik. A new explicit finite free resolution of ideals generated by monomials in an $R$-sequence. J. Pure Appl. Algebra, 51(1-2):193-195, 1988.

[23] Saunders Mac Lane. Homology. Classics in Mathematics. Springer-Verlag, Berlin, 1995. Reprint of the 1975 edition.

[24] Saunders Mac Lane and Garrett Birkhoff. Algebra. Chelsea Publishing Co., New York, third edition, 1988.

[25] J. Peter May. The cohomology of restricted lie algebras and of hopf algebras. J. Alg., 3:123-146, 1966. 
[26] E. Sköldberg. More on the homology of associative algebras, 1995. Preprint, Stockholm University.

[27] D. Taylor. PhD thesis, 1960. Univ. Chicago.

This article may be accessed via WWW at http://www.rmi.acnet.ge/hha/ or by anonymous ftp at

ftp://ftp.rmi.acnet.ge/pub/hha/volumes/2002/n2a14/v4n2a14.(dvi,ps,pdf)

Leif Johansson leifj@matematik.su.se

Mathematics Department

Stockholm University 10691 Stockholm

Sweden

Larry Lambe 1.lambe@bangor.ac.uk

School of Informatics

Division of Mathematics

University of Wales, Bangor

Dean Street

Bangor, Gwynedd LL57 1UT, UK

Emil Sköldberg emil@matematik.su.se

Mathematics Department

Stockholm University 10691 Stockholm

Sweden 